ARTICLE

Received 15 Nov 2016 | Accepted 17 May 2017 | Published 4 Jul $2017 \quad$ DOl: 10.1038/ncomms16050 OPEN

\title{
Norrin-induced Frizzled4 endocytosis and endo-lysosomal trafficking control retinal angiogenesis and barrier function
}

Chi Zhang ${ }^{1}$, Maria B. Lai ${ }^{1}$, Lavan Khandan ${ }^{1}$ Lindsey A. Lee ${ }^{1}$, Zhe Chen ${ }^{1}$ \& Harald J. Junge ${ }^{1}$

\begin{abstract}
Angiogenesis and blood-brain barrier formation are required for normal central nervous system (CNS) function. Both processes are controlled by Wht or Norrin (NDP) ligands, Frizzled (FZD) receptors, and $\beta$-catenin-dependent signalling in vascular endothelial cells. In the retina, FZD4 and the ligand NDP are critical mediators of signalling and are mutated in familial exudative vitreoretinopathy. Here, we report that NDP is a potent trigger of FZD4 ubiquitination and induces internalization of the NDP receptor complex into the endo-lysosomal compartment. Inhibition of ubiquitinated cargo transport through the multivesicular body (MVB) pathway using a dominant negative ESCRT (endosomal sorting complexes required for transport) component VPS4 EQ strongly impairs NDP/FZD4 signalling in vitro and recapitulates CNS angiogenesis and blood-CNS-barrier defects caused by impaired vascular $\beta$-catenin signalling in mice. These findings provide evidence for an important role of FZD4 endocytosis in NDP/FZD4 signalling and in CNS vascular biology and disease.
\end{abstract}

\footnotetext{
${ }^{1}$ Department of Molecular, Cellular, and Developmental Biology, University of Colorado, Boulder, Colorado 80309, USA. Correspondence and requests for materials should be addressed to H.J.J. (email: harald.junge@colorado.edu).
} 
W $\mathrm{nt} / \beta$-catenin signalling (also referred to as canonical Wnt signalling) controls major developmental processes and is implicated in several human diseases, including vascular diseases ${ }^{1}$. Angiogenesis and blood-CNS barrier formation enable CNS function and require $\beta$-catenin signalling in vascular endothelial cells ${ }^{2-6}$. Consequently, impaired blood vessel development and vascular dysfunction are implicated in inherited CNS vascular diseases and neurodegenerative diseases ${ }^{7}$. In the retinal vasculature, canonical signalling is mediated by the ligand NDP and a receptor complex composed of FZD4, LRP5 (low-density lipoprotein receptor-related protein 5), and the tetraspanin family member TSPAN12 (refs 8,9). The crystal structures of NDP in complex with FZD4 show how NDP interacts with FZD4 (refs 10,11) and genetic experiments demonstrate that NDP/FZD4 signalling is mediated by $\beta$-catenin ${ }^{6}$. Mice with gene disruptions in $N d p, F z d 4, L r p 5$ or Tspan12 display overlapping retinal vascular phenotypes characterized by failure of intraretinal blood vessel development and blood-retina barrier defects ${ }^{9,12-15}$. In humans, mutations in NDP, FZD4, LRP5 or TSPAN12 genes are linked to familial exudative vitreoretinopathy (FEVR), which is characterized by retinal vascular defects, vision impairment, or blindness ${ }^{16}$. Thus, $\mathrm{NDP} / \mathrm{FZD} 4$ signalling plays a major role in retinal physiology and in human disease.

Mechanisms of canonical signalling have been elucidated in the context of Wnt-induced B-catenin signalling. Wnt-activated FZD receptor and LRP5/6 co-receptor inhibit glycogen synthase kinase 3 beta (GSK3 $\beta$ ), the central effector of the $\beta$-catenin destruction complex, and thus signal the stabilization of $\beta$-catenin, which in turn forms a transcriptional complex with T-cell factor/lymphoid enhancer factor $(\mathrm{TCF} / \mathrm{LEF})^{17}$. Canonical signalling is coupled to the formation of LRP6-signalosomes, plasma membrane associated aggregates containing phospho-LRP6, dishevelled (DVL), FZD, axin, and other destruction complex components ${ }^{18}$, which require cellular synthesis of 4,5-bisphosphates for assembly ${ }^{19}$. Clathrin and the endocytic adapter complex AP2, which interacts with $\mathrm{DVL}^{20}$, have been reported to promote signalosome formation and signalling ${ }^{21,22}$.

LRP6 endocytosis has been studied both as a mechanism of desensitization ${ }^{23,24}$ and as mediator of a component of signalling that originates from the endo-lysosomal compartment ${ }^{25-28}$. The ligand-induced internalization of receptor, co-receptor and associated destruction complex components are thought to lead to productive signalling from endosomes and/or to sequestration of GSK3 $\beta$ into MVBs, thus preventing GSK3 $\beta$ from marking cytoplasmic $\beta$-catenin for degradation ${ }^{29,30}$. This mechanism, next to the direct inhibition of GSK3ß by the activated receptor complex, may lead to enhanced or sustained signalling ${ }^{31}$. Consistent with a role of the endo-lysosomal compartment in canonical signalling is the finding that endosomal acidification by V-ATPase, which is physically linked to the receptor complex via the prorenin receptor, is a requirement of $\mathrm{Wnt} / \beta$-catenin signalling ${ }^{32}$. GSK $3 \beta$ sequestration requires the function of ESCRT complexes, which mediate the movement of ubiquitinated cargo into late endosomes. However, FZD ubiquitination has not been reported to be ligand dependent ${ }^{33}$ and is not known to lead to GSK3 $\beta$ sequestration. Instead, in the absence of WNTs, FZD ubiquitination by ZNRF3 and RNF43, two homologous transmembrane E3 ubiquitin ligases that ubiquitinate FZD receptors, induces FZD clearance from the plasma membrane to diminish signalling ${ }^{34-37}$.

FZD endocytosis has been predominantly studied in the context of non-canonical signalling. FZD4 endocytosis triggered by a combination stimulus WNT5A and PKC activator PMA (phorbol 12-myristate 13-acetate) is DVL-dependent and has been linked to planar cell polarity signalling ${ }^{38}$. In studies of canonical signalling, reports have focused on interactions of the endocytic machinery with LRP6 or DVL, whereas little is known about ligand-induced FZD interactions with mediators of internalization.

Here, we investigate the role of endocytosis in the canonical NDP/FZD4 signalling system. We find that NDP promotes FZD4 ubiquitination and potently triggers internalization of the NDP receptor complex components FZD4, LRP5 and TSPAN12 into the endo-lysosomal compartment. Inhibition of the MVB pathway using dominant negative VPS4 EQ strongly impairs NDP/FZD4 signalling in vitro. Induced expression of VPS4 EQ in vascular endothelial cells in mice recapitulates previously reported angiogenesis and blood-CNS barrier defects caused by endothelial cell-specific B-catenin deletion. We conclude that NDP-induced FZD4 endocytosis and endo-lysosomal trafficking control retinal angiogenesis and barrier function.

\section{Results}

NDP triggers the internalization of its receptor complex. To determine if NDP triggers endocytosis of the NDP-receptor complex, we transfected HeLa cells with V5-FZD4, LRP5 and HA-TSPAN12. After incubation of the cells with alkaline phosphatase (AP)-tagged NDP conditioned medium on ice, unbound NDP was removed and endocytosis enabled at $37^{\circ} \mathrm{C}$. Cells displayed both surface-bound and internalized NDP in the absence of an acid wash step before fixation (Fig. 1a), whereas washing with acidic buffer removed surface-bound NDP and revealed specifically internalized NDP (Fig. 1b; Supplementary Fig. 1a). Transfected FZD4 was essential for both cell surface binding and internalization of NDP, while LRP5 and TSPAN12 were not sufficient to mediate either process (Fig. 1a insets, see also untransfected cells in Supplementary Fig. 1a). FZD4 was detected predominantly at the cell surface in cells subjected to the internalization protocol in the absence of NDP (Fig. 1c), however, addition of NDP triggered the accumulation of FZD4 in intracellular puncta (Fig. 1d). When cells were incubated with both NDP and a V5-antibody targeting the extracellular tag of V5-FZD4, internalized antibody revealed specifically intracellular FZD4 after acid wash (Fig. 1e). Co-transfection efficiency in HeLa cells was very high (Supplementary Fig. 1b). When all receptor complex components were co-transfected, internalized NDP co-localized with each component of the receptor complex, that is, FZD4, TSPAN12 and LRP5, indicating that the entire receptor complex was endocytosed (Fig. 1f-h). We used the previously described FZD4 M157V mutation ${ }^{39}$ in the binding interface for $\mathrm{NDP}^{10,11}$ to impair NDP binding. When the NDP/FZD4 interaction was disturbed using the FZD4 M157V mutation, NDP-induced endocytosis was clearly reduced (Supplementary Fig. 1c-g).

Internalized NDP and FZD4 in the endo-lysosomal compartment. To reveal the destination of the internalized complex, we performed co-localization experiments with markers for endosomal and lysosomal compartments, that is, EEA1 (early endosome) RAB11 (recycling endosome), RAB7 (late endosome) and LAMP1 (late endosome and lysosome). FZD4 puncta were positive for EEA1 15-30 min after induction of endocytosis. At the 60-min time point, FZD4 co-localized in part with EEA1 and in part with RAB7 and LAMP1; virtually no co-localization with RAB11 was detected (Fig. 2a-d). NDP and FZD4 together co-localized with endosomal and lysosomal markers, indicating that receptor and ligand are targeted to the same destination after internalization (Fig. 2e,f).

TSPAN12 and LRP5/6 are not required for FZD4 endocytosis. We next investigated whether the receptor complex components 


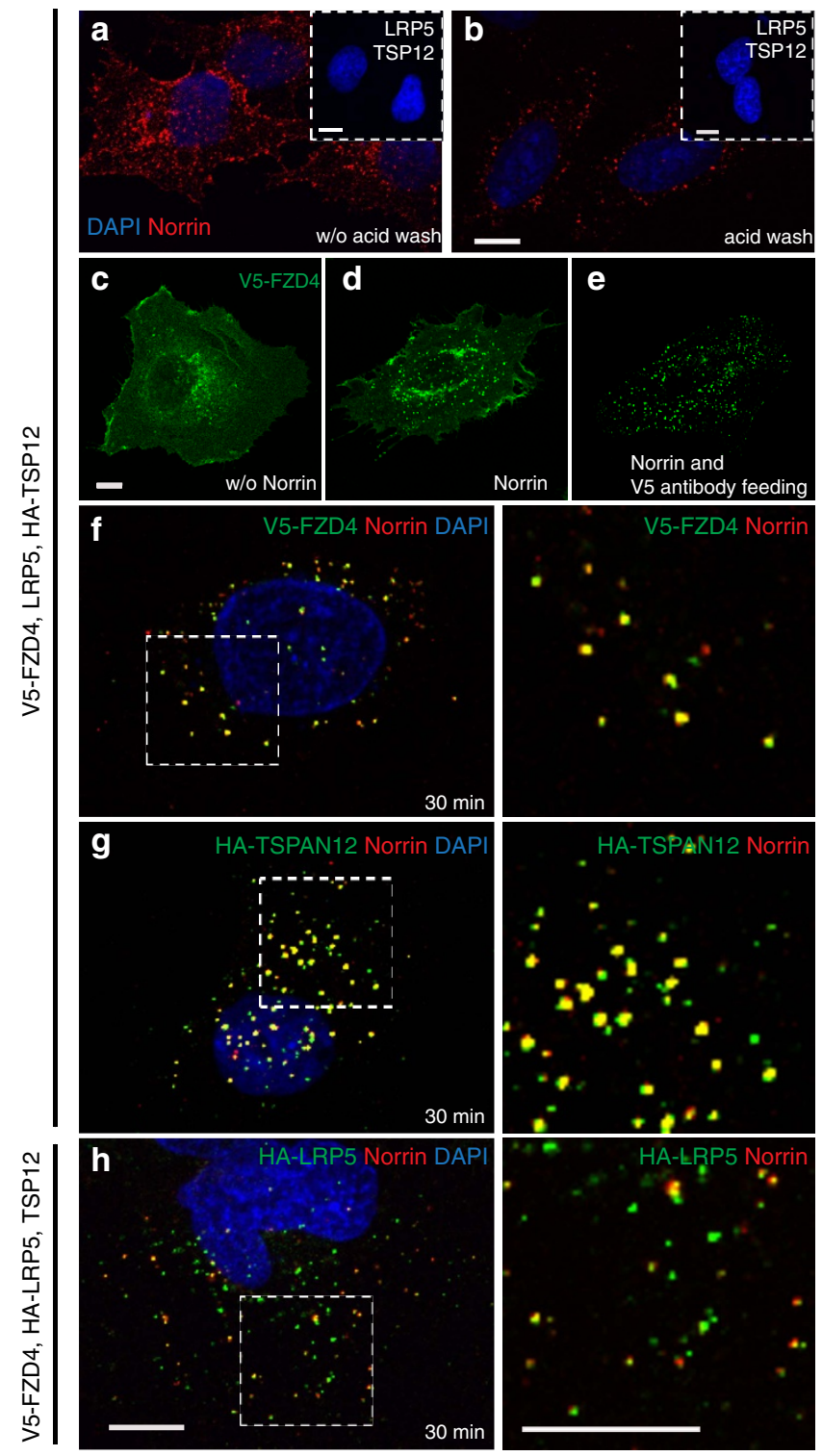

Figure 1 | Norrin induces endocytosis of the FZD4 receptor and associated membrane proteins. (a) HeLa cells incubated with AP-Norrin-mycHis conditioned medium on ice were washed and then subjected to a $30 \mathrm{~min} 37^{\circ} \mathrm{C}$ stimulus to induce endocytosis. Staining reveals both cell surface bound and internalized Norrin (via myc). Inset: transfection of co-receptor LRP5 and co-activator TSPAN12 is not sufficient to mediate binding or internalization of Norrin. (b) Removing surface bound Norrin by an acid wash step reveals specifically internalized Norrin.

(c) FZD4 shows predominantly cell surface localization in the absence of Norrin. (d) Norrin-induced FZD4 internalization in a cell stained after permeabilization. (e) V5-FZD4 at the cell surface of intact cells was labelled with a V5 antibody. Induction of endocytosis with Norrin and subsequent acid wash allows to specifically reveal internalized FZD4 via the co-internalized V5 antibody ('antibody feeding'). (f-h). Norrin and FZD4 co-localize in endocytic puncta. Co-activator TSPAN12 and co-receptor LRP5 are co-internalized. Boxed areas in $\mathbf{f}-\mathbf{h}$ are shown enlarged on the right with the DAPI channel omitted. Internalized FZD4 is specifically revealed by $\mathrm{V} 5$ antibody feeding. A similar experiment without V5 antibody feeding is shown in Supplementary Fig. 1a. Scale bars, $10 \mu \mathrm{m}$.

LRP5/6 or TSPAN12 are required for NDP-induced FZD4 internalization. We used CRISPR/Cas9 mediated genome targeting to generate HeLa cells deficient for both LRP5 and LRP6 and a separate HeLa cell line deficient for TSPAN12
(Supplementary Fig. 2; Supplementary Fig. 3; Supplementary Fig. 4). LRP5 $5^{-1-}$; LRP6 ${ }^{-I-}$ HeLa cells or TSPAN12-I- HeLa cells transfected with FZD4 efficiently internalized NDP into early endosomes (Supplementary Fig. 5a-d). These results indicate that FZD4 is a critical mediator of NDP-induced receptor complex internalization. Although LRP5/6 interacts independently with the endocytic machinery ${ }^{27}$, FZD4 is sufficient to mediate NDP-induced internalization. However, FZD4 alone (in the absence of LRP5/6) is not sufficient to elicit signalling ${ }^{6,9,12}$.

NDP-induced FZD4 endocytosis does not require dishevelled. Previous studies showed that the combination stimulus of WNT5A and the phorbol ester PMA triggers FZD4 endocytosis ${ }^{40}$. DVL2 binding to AP2 is essential for this endocytic process, which has been linked to non-canonical signalling ${ }^{38}$. We asked if NDP-induced FZD4 endocytosis also requires DVL2. First, we confirmed that we could trigger FZD4 endocytosis using WNT5A/PMA (Supplementary Fig. 6). We then used previously reported mutations, that is, the $\mathrm{K} 466 \mathrm{M}$ mutation in the DEP domain of DVL to disrupt binding to FZD4, and the DVL2 AHEL mutation to disrupt binding to the AP2 $\mu 2$ subunit $^{20,38}$. We monitored FZD4/DVL2 binding using a DVL aggregation assay $^{41}$. DVL2 formed cytoplasmic aggregates when overexpressed in the absence of FZD4, however, when FZD4 was co-expressed with DVL, aggregates did not form and DVL was efficiently recruited to the plasma membrane (Supplementary Fig. $7 c, d)$. As expected, DVL2 AHEL was recruited to FZD4 in a similar manner as WT DVL2, however, DVL2 K466M remained aggregated in the cytoplasm (Fig. 3, right panels). Both DVL2 AHEL and K466M mutations interfered with WNT5A/ PMA-triggered FZD4 endocytosis (Fig. 3a-c). Interestingly, NDP-induced FZD4 endocytosis was not impaired by DVL2 mutations K466M or AHEL (Fig. 3d-f). To further test the requirement of FZD4/DVL2 binding, we mutated residues in the intracellular domain of FZD4 that are predicted to interact with DVL2 based on studies of other Frizzleds. One FZD/DVL interaction site is the KTxxxW motif in the c-terminal tail of FZD4 (ref. 42), and additional interaction sites in intracellular loops have been identified in FZD5 (ref. 41). These studies enabled us to identify a triple mutation - FZD4 I416A, L430A, K499A - that failed to re-localize DVL2 from cytoplasmic aggregates to the plasma membrane. In addition, we found that a FEVR mutation, FZD4 W496X (ref. 43), disrupted DVL2 binding (Supplementary Fig. 7). FZD4 W496X and FZD4 I416A, L430A, K499A failed to internalize in response to WNT5A/PMA stimulation (Fig. $4 \mathrm{a}-\mathrm{c}$ ). In clear contrast, these FZD4 mutations did not interfere with NDP-induced FZD4 endocytosis (Fig. 4d-f).

NDP-induced FZD4 ubiquitination triggers endocytosis. To test if NDP-induced FZD4 endocytosis requires FZD4 ubiquitination, we used FZD4 K13R (all intracellular lysine residues replaced with arginine, also referred to as K0) and FZD4 K12R (residue 499, which is important for DVL2 binding, is intact). Dishevelled aggregation assays showed that FZD4 K12R retains binding to DVL2 and surface biotinylation assays showed that FZD4 K12R cell surface expression was moderately increased (Supplementary Fig. 8a,b). NDP failed to induce substantial FZD4 endocytosis in cells expressing FZD4 K12R or FZD4 K13R. While the impairment of endocytosis was strong and obvious, inspection of cells in detail and quantification of internalized Norrin positive puncta revealed that endocytosis was not completely abolished (Fig. 5a,b). This mechanism of FZD4 endocytosis was surprising, as FZD4 ubiquitination was previously implicated in regulating receptor clearance, but not in ligand-induced FZD endocytosis ${ }^{33,34,36}$. To test if NDP triggers 


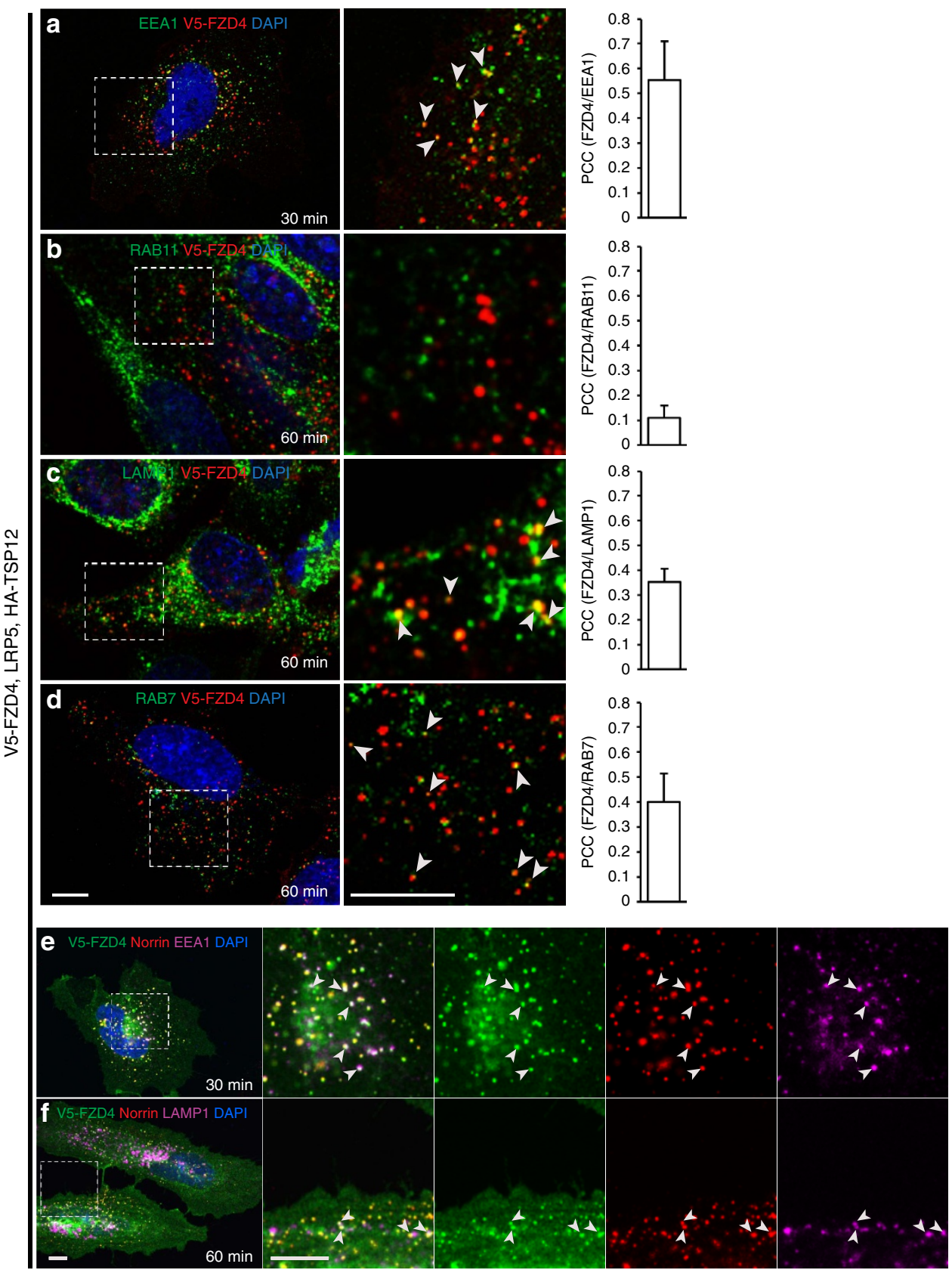

Figure 2 | Internalized FZD4 is targeted to the endosome and lysosome. (a) 30 min after Norrin-induced internalization at $37^{\circ} \mathrm{C}$ FZD 4 co-localizes with the early endosomal marker EEA1 in HeLa cells. (b) No substantial co-localization with the recycling endosome marker Rab11 was detected. (c) FZD4 co-localizes with the lysosomal marker LAMP1 and (d) RAB7. Co-localization was quantified using the Pearson correlation coefficient. Mean + s.d. shown, $n=20$. In a-d internalized FZD4 is specifically revealed by co-internalized V5-antibody. (e,f) Triple staining for FLAG-AP-Norrin, V5-FZD4 and markers for membrane trafficking excited in the $488 \mathrm{~nm}$ (green), $555 \mathrm{~nm}$ (red) and $647 \mathrm{~nm}$ (magenta) channels. Boxed areas are shown enlarged on the right. The DAPI channel was omitted in the enlarged 3-colour merge. Norrin and FZD4 traffic together into the early endosome and lysosome. Arrowheads show examples of co-localization. Scale bars, $10 \mu \mathrm{m}$.

FZD4 ubiquitination, HeLa cells were co-transfected with HA-ubiquitin and V5-FZD4. The intact cells were labelled with V5 antibody on ice and cell surface FZD4 was subsequently isolated using Protein A/G beads. To test the role of NDP in promoting ubiquitination, NDP conditioned medium or control conditioned medium was added together with the V5 antibody and cells were subjected to $20 \mathrm{~min}$ internalization at $37^{\circ} \mathrm{C}$. NDP-induced a substantial increase of FZD4 ubiquitination, which was blocked by both K12R and K13R mutations (Fig. 5c). To confirm that the mechanisms of FZD4 endocytosis are recapitulated in endothelial cells, we used a CNS endothelial cell line, bEND.3. Internalized NDP co-localized with FZD4, EEA1 and LAMP1, and internalization was prevented by K12R mutations in FZD4 (Supplementary Fig. 8c-g). Together, our results reveal that NDP promotes both FZD4 ubiquitination and FZD4 internalization.

Blockade of endo-lysosomal trafficking inhibits signalling. To determine the functional role of endocytosis and endo-lysosomal trafficking in NDP/FZD4 signalling, we blocked these processes using inhibitors and dominant negative interference, and examined the resulting signalling responses using the TOPFlash 

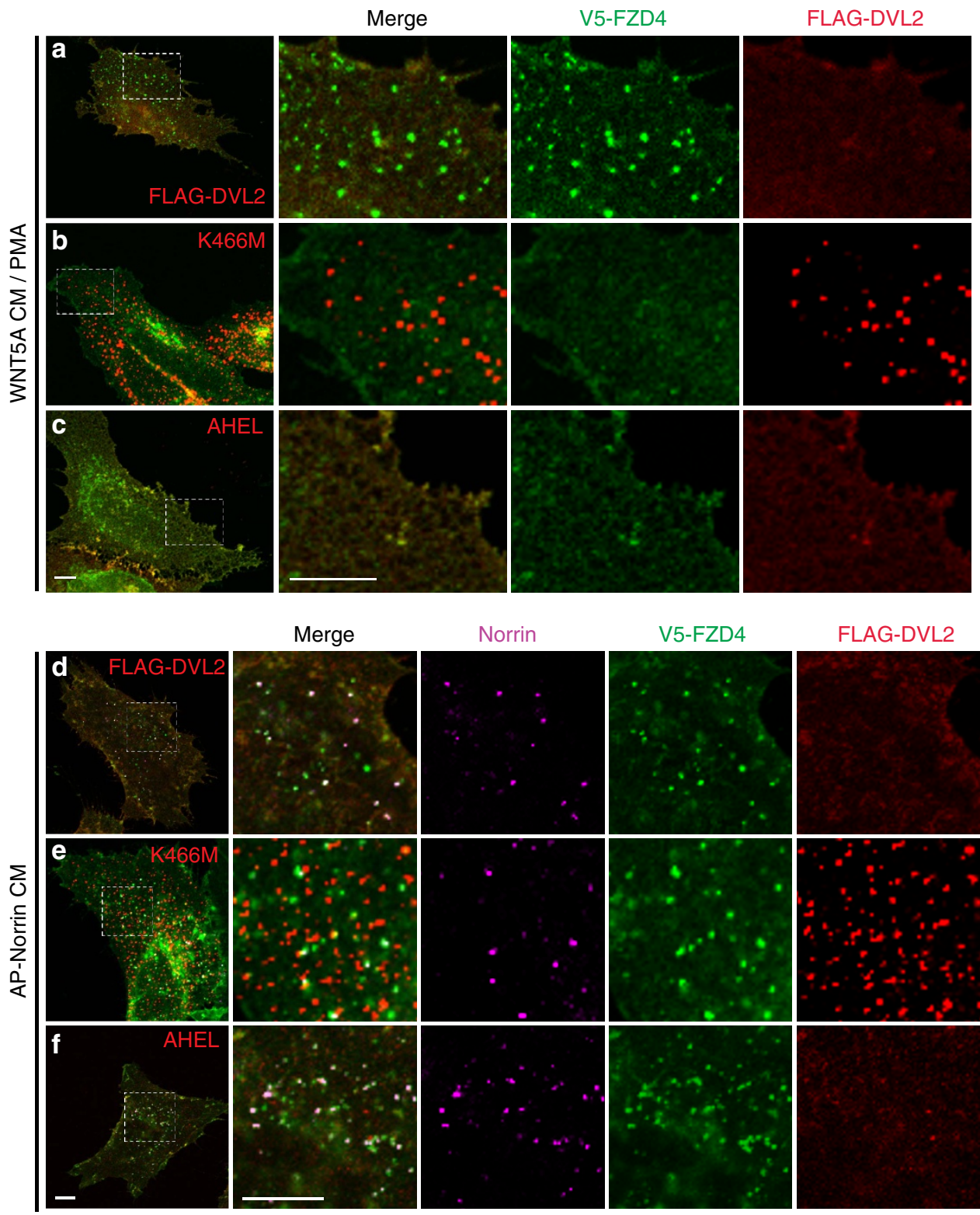

Figure 3 | Effect of DVL2 mutations on FZD4 endocytosis. (a) WNT5A/PMA-induced FZD4 internalization in HeLa cells. WT DVL2 binds and co-localizes with FZD4 and does not form cytoplasmic aggregates. (b) DVL2 K466M is FZD4-binding deficient and thus forms cytoplasmic aggregates. In the DVL2 K466M expressing cells, WNT5A/PMA fails to induce FZD4 endocytosis. (c) DVL2 AHEL, which is impaired in AP2 binding, does not form cytoplasmic aggregates but also prevents WNT5A/PMA-induced internalization of FZD4. (d-f) NDP induces strong FZD4 endocytosis regardless of K466M or AHEL mutations in co-expressed DVL2. Scale bar, $10 \mu \mathrm{m}$.

reporter assay ${ }^{44}$. Dynasore is a small molecule inhibitor of dynamin, a GTPase that is required for clathrin- and caveolindependent endocytosis. We found that dynasore inhibited NDP-induced signalling in a concentration-dependent manner. TSPAN12 is a ligand-specific co-activator in NDP/FDZ4 signalling 9 , and Dynasore did not affect TSPAN12-mediated enhancement of signalling relative to the base level (Fig. 6a). We investigated potential crosstalk of NDP with endogenous WNTs and found that (i) NDP and WNT7B did not show additive effects in TOPFlash assays, (ii) NDP-induced signalling was not blocked by the combination of two WNT inhibitors, sFRP1 and IWP-2, and (iii) Dynasore inhibited NDP-induced signalling even when endogenous WNTs were inactivated by sFRP1 and IWP-2 treatment (Supplementary Fig. 9a-c). These results confirmed that Dynasore affected NDP/FZD4 signalling in a largely Wnt-independent manner.
ESCRT complexes function in the MVB pathway to deliver ubiquitinated membrane proteins to the late endosome. Multiple components of ESCRT-0 and ESCRT-I complexes bind ubiquitinated cargo via ubiquitin interacting motifs. The ATPase VPS4 is an essential mediator of MVB formation by disassembling ESCRT-III complexes and recycling individual components for subsequent rounds of vesicle formation ${ }^{45}$. Consistent with previous reports ${ }^{30}$, we found that the co-transfection of the ATPase VPS4b (with c-terminal mycHis tag) did not affect NDP/FZD4 signalling, however, the dominant negative VPS4b $\mathrm{EQ}$, that is, E235Q in mouse VPS4b (ref. 46), strongly inhibited signalling (Fig. 6b). On the subcellular level, VPS4 EQ affected NDP trafficking through the endo-lysosomal compartment. $3.5 \mathrm{~h}$ after induction of endocytosis, NDP had moved predominantly to the LAMP1-positive compartment in VPS4 expressing cells, whereas NDP still substantially co-localized with EEA1 and 

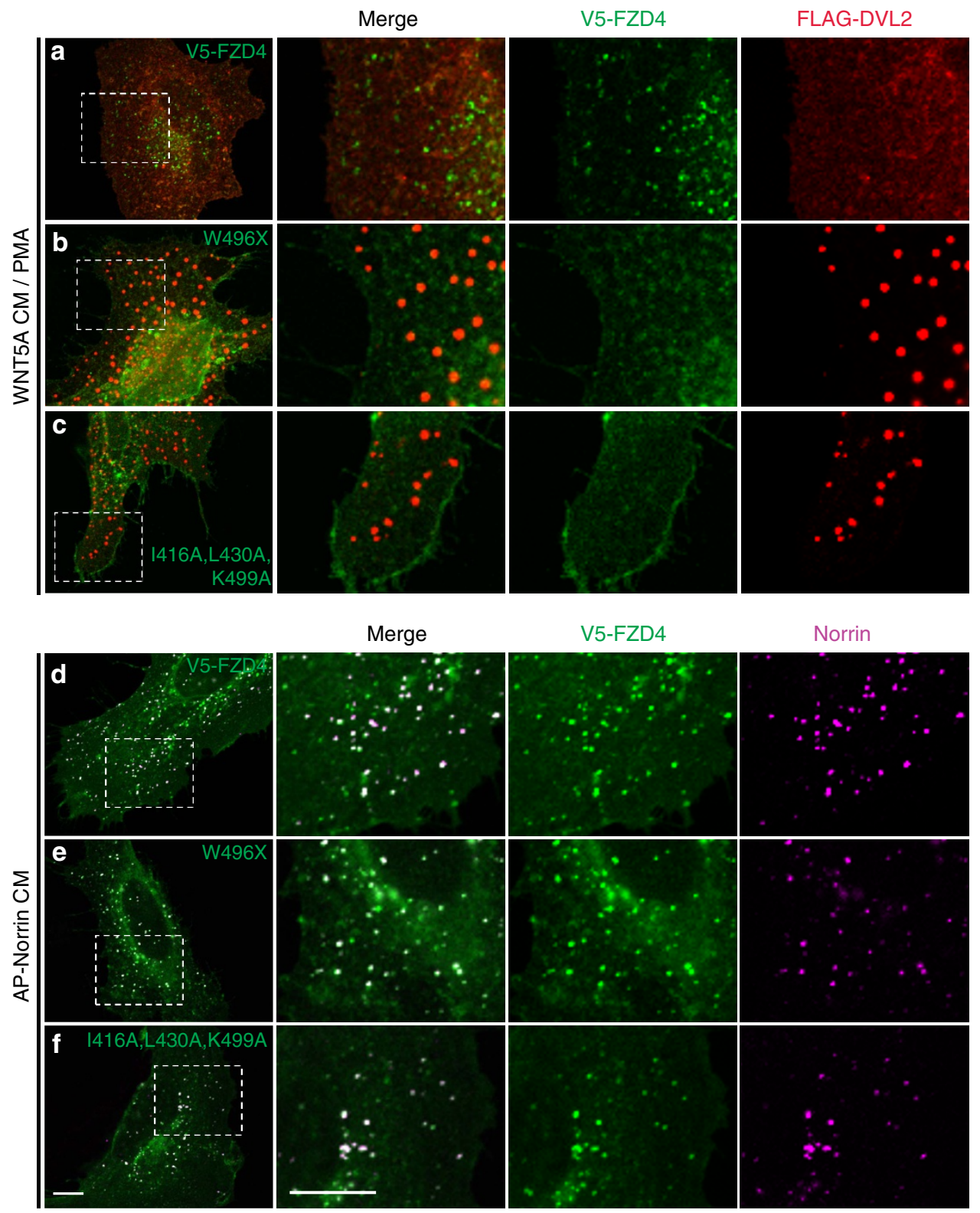

Figure 4 | DVL2 binding to FZD4 is dispensable for Norrin-induced endocytosis. (a-c) FZD4 mutations that disrupt DVL2 binding fail to recruit DVL2 to the plasma membrane and block WNT5A/PMA-induced endocytosis. (d-f) Norrin-induced FZD4 endocytosis is not affected by FZD4 mutations that disrupt DVL2 binding. DVL2 was co-expressed in all a-f. Scale bar, $10 \mu \mathrm{m}$.

showed minor co-localization with LAMP1 in VPS4 EQ expressing cells (Fig. $6 \mathrm{c}-\mathrm{h}$ ). Together, these data indicate that impaired trafficking through the MVB pathway and NDP/FZD4 signalling defects are associated.

Angiogenesis and barrier defects in VPS4 EQ expressing mice. To further test the requirement of moving ubiquitinated cargo through the MVB pathway in NDP/FZD4 signalling, we constructed a Cre-activated knock-in allele that encodes murine VPS4 EQ and targeted it to the Rosa26 locus (Supplementary Fig. 10). In this allele, the CAG promoter is separated from the open reading frame by multiple transcriptional stop signals, which in turn are flanked by loxP sites (loxP-STOP-loxP, LSL). We used an endothelial cell specific Cre-ERT2 driver line, $\mathrm{Tg}$ (Cdh5-cre/ERT2)1Rha (ref. 47) to induce VPS4 EQ expression by tamoxifen injection at P6 and P7. At P9, robust VPS4 EQ expression was detected in lysates of lung tissue, which was used because of its high vascular density (Supplementary Fig. 10c). Analysis of P19 sagittal brain sections revealed widespread leakage of Sulfo-NHS-biotin tracer in VPS4 EQ activated mice, including in the cortex, hippocampus, and molecular layer of the cerebellum (Fig. 7a,b). P10 retinal sections of VPS4 EQ activated mice displayed the characteristic endothelial cell PLVAPhigh expression signature and showed that the formation of intraretinal blood vessels had failed (Fig. 7c). P10 whole mount retinas were analyzed using confocal microscopy and showed that VPS4 EQ expressing mice displayed additional characteristic loss-of-function phenotypes indicative of impaired NDP/FZD4 signalling $9,12-15$. The radial vascular growth in the nerve fiber layer was delayed, vascular malformations were detected at the vascular front, and blood vessels failed to invade the outer plexiform layer of the retina. Highly characteristic glomeruloid vascular malformations formed instead of intraretinal capillaries (Fig. $7 \mathrm{~d}-\mathrm{g}$ ). VPS4 EQ expression resulted in sudden lethality 4-5 days after recombination, reminiscent of the lethality 
a

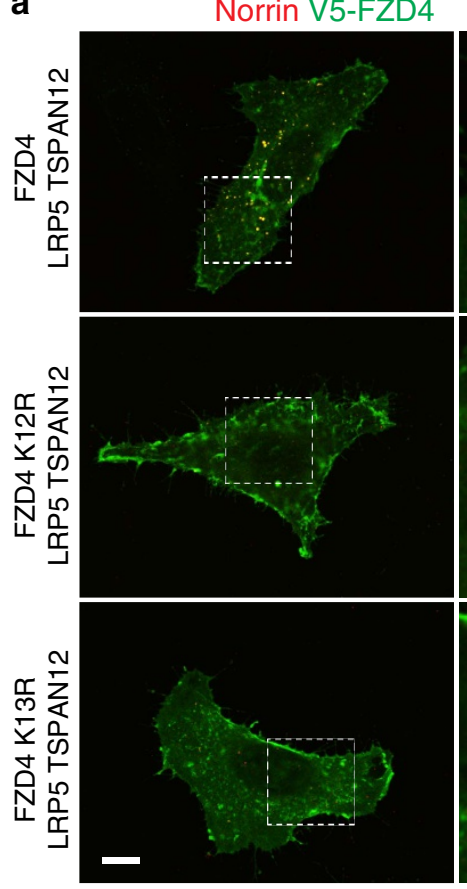

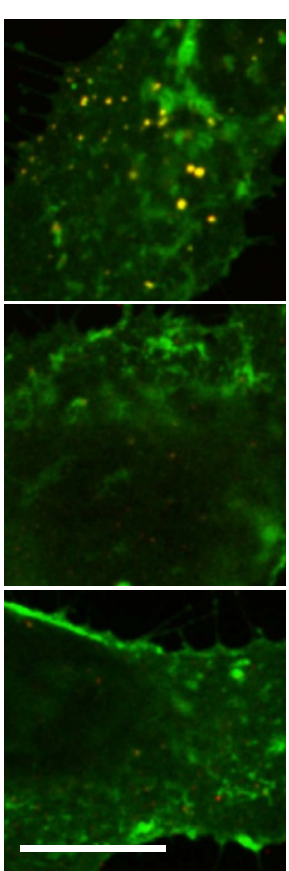

b

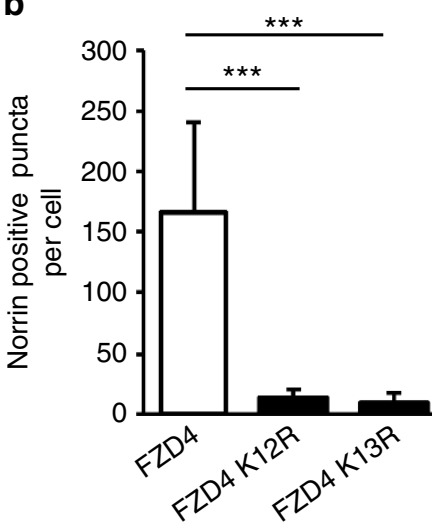

C
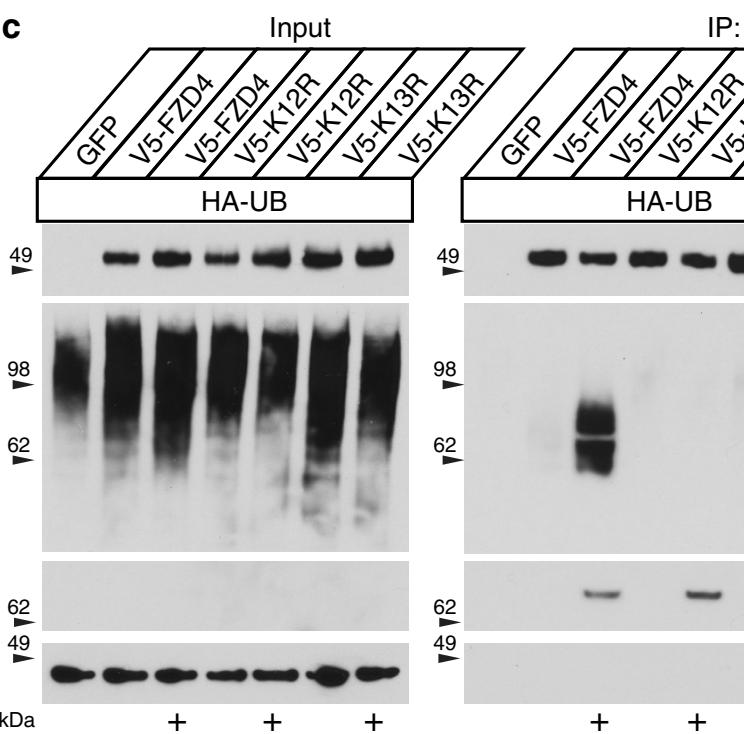

IP: V5 
a

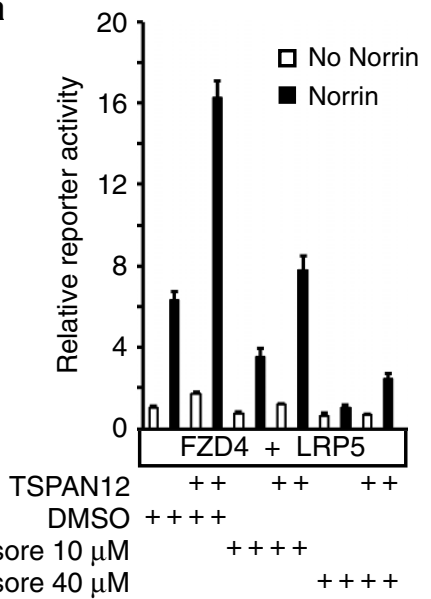

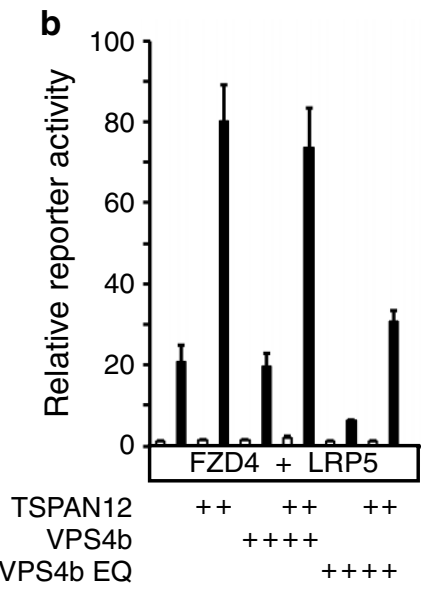
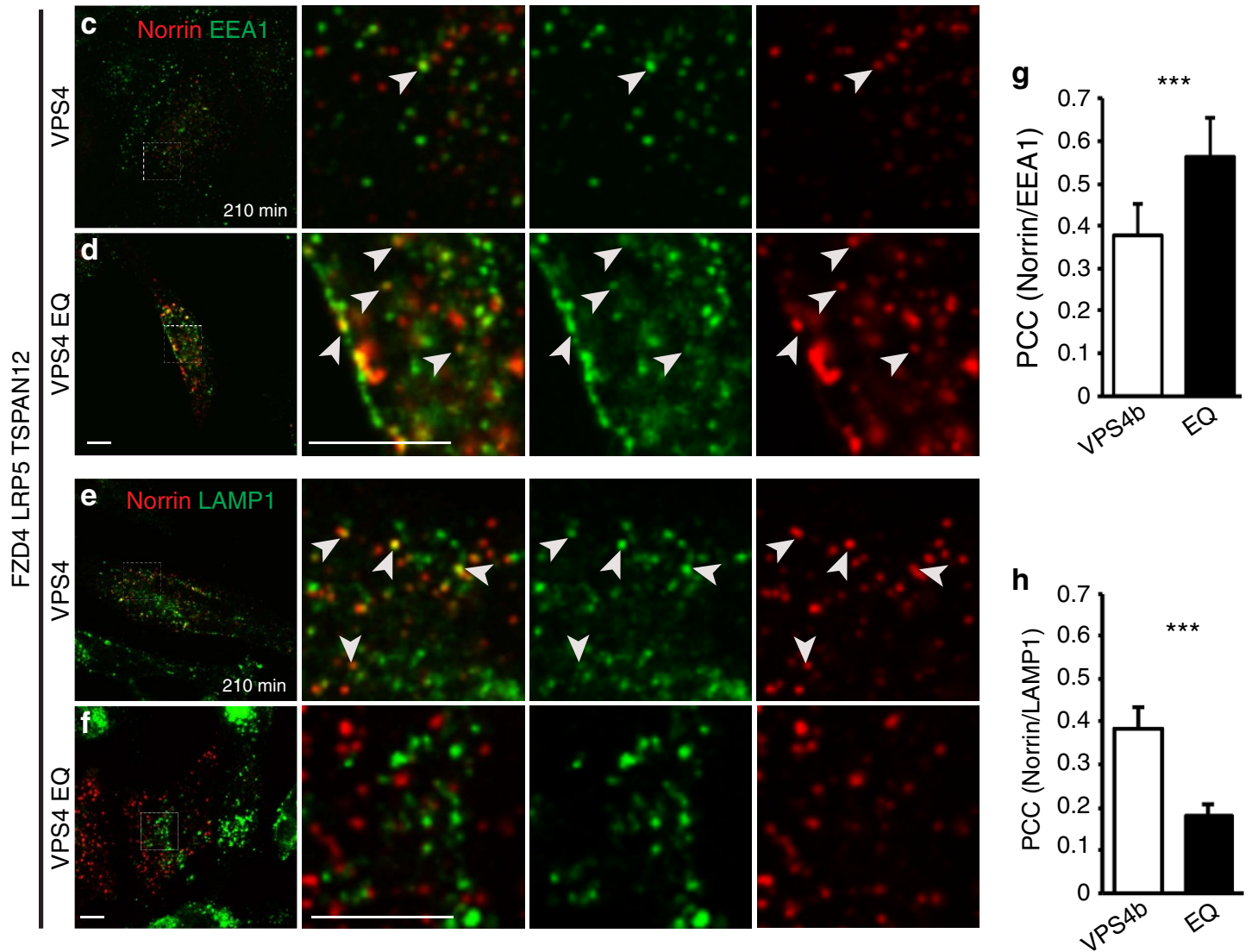

Figure 6 | Inhibition of endo-lysosomal trafficking impairs FZD4 signalling. (a) TOPFlash reporter assays in 293T cells transfected with the indicated constructs show that Dynasore inhibits Norrin/FZD4 signalling in a dose-dependent manner. (b) Co-expression of WT VPS4 has no effect on Norrin/FZD4 signalling, whereas dominant negative VPS4 EQ impairs Norrin/FZD4 signalling. Data are normalized to the first bar in each graph $(n=3$, mean + s.d. shown). Different signalling strength in $\mathbf{a}$ versus $\mathbf{b}$ is due to a shorter stimulation time in experiments where dynasore was added. (c-f) $3.5 \mathrm{~h}$ after induction of endocytosis, Norrin co-localized predominantly with LAMP1 in VPS4 expressing HeLa cells, and predominantly with EEA1 in VPS4 EQ expressing cells. In VPS4 EQ expressing cells, Norrin accumulation in early endosomes produced occasionally unusually large fluorescent puncta. (g,h) Quantification of co-localization $\left(n=20\right.$, mean + s.d. shown, $\left.{ }^{\star \star \star} P<0.001\right)$. Scale bar, $10 \mu \mathrm{m}$.

diseases associated with an impaired blood-brain barrier, may be linked to dysfunctional endo-lysosomal trafficking.

We further show that the NDP/FZD4 ligand-receptor pair provides a suitable model system to study FZD internalization in $\beta$-catenin-dependent signalling. A challenge to biochemical and cell biological studies using Wnts is the promiscuous interaction of Wnt ligands with multiple FZD receptors and the requirement for lipid modifications, which enable the secretion of $\mathrm{Wnts}^{48}$ but make handling Wnts more difficult. In comparison, NDP signals specifically through FZD4, is genetically well characterized, and does not require lipid modification. Both internalized ligand and receptor can be readily localized within cells. Thus, NDP/FZD4 signalling provides a system to investigate mechanisms of ligand-induced FZD internalization in canonical signalling.

Previous studies on ligand-induced receptor internalization have predominantly focused on the ligands WNT5A in 

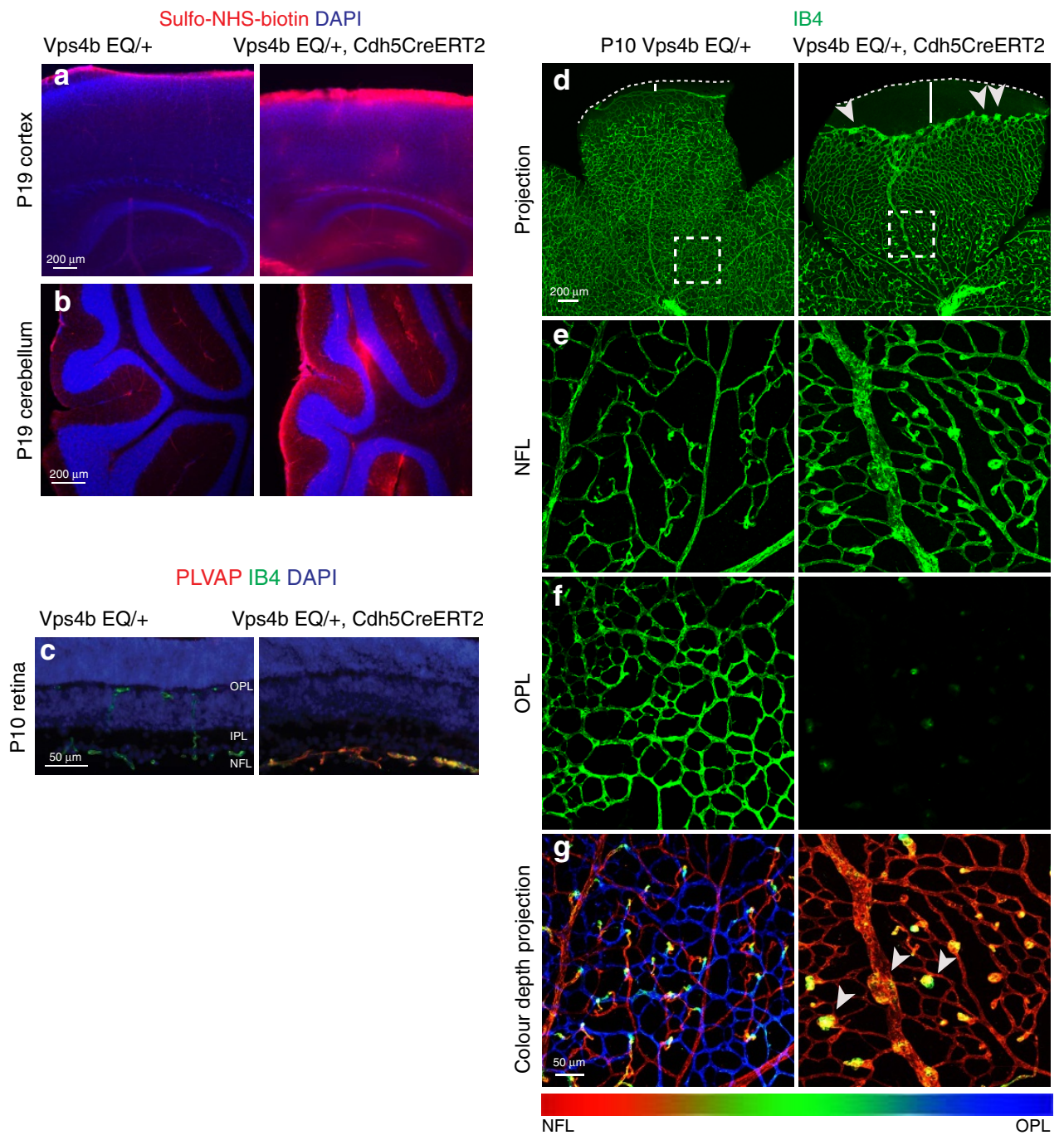

Figure 7 | Inhibition of endo-lysosomal trafficking in vascular endothelial cells in vivo. (a) Blood-CNS barrier defects are revealed in the cortex and hippocampus of Cre-activated Rosa26 LSL-VPS4 EQ mice transcardially perfused with Sulfo-NHS-biotin by staining with Streptavidin coupled to Alexa 555. (b) Barrier defects in the molecular layer of the cerebellum. (c) Retinal sections show failure of intraretinal vascularization and strongly increased PLVAP expression in VPS4 EQ expressing mice. These defects recapitulate hallmark phenotypes of impaired Norrin/FZD4 signalling in the retinal vasculature. NFL, nerve fiber layer; IPL, inner plexiform layer; OPL, outer plexiform layer. (d) Confocal projections of the retinal vasculature in P10 whole mount retinas stained with IsolectinB4-Alexa488. Characteristic Norrin LOF phenotypes are observed. Progression towards the rim of the retina (indicated by white dashed line) is delayed (distance from the vascular front to the rim of the retina indicated by white bar) and the microscopic appearance of the vascular front is altered (arrowheads). (e,f) Optical sections of the boxed areas in $\mathbf{d}$ show separate representations of the vascular plexuses located at different depth levels of the retina. Normal vasculature is present in the nerve fiber layer (NFL) and outer plexiform layer (OPL) in control mice, whereas intraretinal capillaries in the OPL of VPS4 EQ expressing mice are absent. (g) Colour depth projections show the vascular beds of the boxed areas in $\mathbf{d}$ as single projection, in which distinct depth levels are colour coded. These projections reveal the characteristic glomeruloid vascular malformations (yellow structures, arrowheads) in the nerve fiber layer vasculature, similar to the defects described in Fzd4, Ndp, Lrp5 and Tspan12 mutant mice.

non-canonical signalling and WNT3A in canonical signalling. We find that NDP-induced FZD4 internalization does not require DVL2/FZD4 binding, whereas WNT5A/PMA-induced FZD4 internalization requires this interaction. Studies on WNT3Ainduced receptor internalization have characterized mechanisms of LRP6 endocytosis ${ }^{27}$, but little is known about interactions of FZD with the endocytic machinery when internalization is induced by canonical ligands. In this study we identified ubiquitinated FZD4 as key interaction partner with the endocytic machinery in NDP-induced internalization. Although FZD4 co-internalizes with LRP5/6, neither LRP5/6 nor DVL are required for internalization. NDP-induced endocytosis of FZD4 without associated LRP5/6 or DVL (for example, in LRP5/6 DKO cells or when FZD4 and DVL cannot interact due to mutations in
FZD4 or DVL) is, however, not sufficient to elicit signalling. FZD and LRP5/6 appear to have separate interactions with mediators of internalization, and the contribution of FZD in the process of ligand-induced endocytosis had so far been unclear.

FZD receptor levels and signalling strength are controlled by E3 ligases ZNRF3 and RNF43, which are in turn modulated by $\mathrm{R}$-spondin ligands and LGR receptors ${ }^{34,36}$. Here, we find that FZD4 ubiquitination is strongly promoted by NDP and leads to NDP-induced endocytosis of FZD4. An important distinction in the regulation of FZD receptor levels and NDP-induced FZD4 internalization is the ability to inhibit the destruction complex. Clearance of FZD in the absence of WNT/NDP does not elicit signalling. In contrast, NDP-induced internalization of a complex containing FZD4, NDP and LRP5 appears to be positively 
coupled to a component of signalling through inhibition of the destruction complex. NDP has been reported to bind LGR4 (ref. 49) and thus, next to its direct actions via FZD4 binding, may also promote FZD signalling in an indirect (that is, R-spondin-like) manner, by removing negative regulators RNF43 and ZNRF3 from the cell surface.

Previous reports indicated that FZD4 ubiquitination is not induced by $\mathrm{WNT}_{3} \mathrm{~A}^{33}$. Ligand properties such as the specific affinity of the ligand for FZD4 (versus other FZDs) may account for different results using NDP versus WNT3A stimulation. In addition, WNT-induced internalization may require additional receptor complex components that stabilize ligand-receptor interactions, a candidate for such a function is GPR124 (ref. 50).

The dominant negative form of VPS4 was previously shown to inhibit Wnt/ $\beta$-catenin signalling in cell-based assays and in frog axis duplication assays ${ }^{30}$. We confirmed and extended these observations in the context of CNS vascular biology. VPS4 is essential for multivesicular body formation and fulfills important roles in sorting ubiquitinated cargo into late endosomes. The blood-brain barrier phenotypes we observed upon activation of dominant negative VPS4 EQ in endothelial cells are similar (albeit probably milder) to those reported for conditional disruption of the $\beta$-catenin gene in endothelial cells ${ }^{6}$. Retinal phenotypes of VPS4 EQ expressing mice are clearly overlapping with defects in mice, in which NDP/FZD4 signalling is disrupted (see Introduction). These striking phenotypic similarities include the characteristic upregulation of the endothelial cell fenestration component PLVAP, delayed progression of the vascular front in the superficial vascular plexus, specific failure of intra-retinal capillary development, and glomeruloid vascular malformations. Impaired endo-lysosomal trafficking could also impair additional signalling systems, for example GSK3 $\beta$ activity that is not mediated by $\beta$-catenin (WNT/STOP signalling ${ }^{51}$ ), or distinct angiogenic signalling systems, for example, Notch, VEGFR2 or $\mathrm{Eph} /$ Ephrin signalling ${ }^{52}$. However, we observed no retinal phenotypes in VPS4 EQ expressing mice that were not also observed in the mouse models with impaired NDP/FZD4 signalling. The specific phenotypic profile in the absence of broader vascular phenotypes suggest that (i) VPS4 EQ activation impairs canonical signalling in a surprisingly specific manner, or (ii), that impaired $\beta$-catenin signalling is one of the earliest consequences of VPS4 EQ activation in our short-term in vivo experiments, or (iii), that impairments in other angiogenic pathways synergize with impaired $\beta$-catenin signalling. This would be expected if the additional impaired pathway mediates the expression of genes that are required for $\beta$-catenin signalling.

Mutations in human genes NDP, FZD4, LRP5 and TSPAN12 cause familial exudative vitreoretinopathy, a potentially blinding disease initially characterized by retinal hypovascularization ${ }^{16}$. Our results suggest a link between defective endo-lysosomal trafficking and FEVR and raise the possibility that mediators of membrane dynamics are additional unknown FEVR genes or modifiers of FEVR expressivity. In neurology, blood-brain barrier defects have been connected to neurodegenerative diseases, including Alzheimer's disease and Parkinson's disease ${ }^{7}$. Thus, in light of our findings, vascular endo-lysosomal trafficking defects may emerge as factors in blood-CNS barrier dysfunction and neurodegenerative diseases.

\footnotetext{
Methods

DNA constructs. Super $8 \mathrm{X}$ TOPFlash ${ }^{44}$ and CMV-Renilla were kindly provided by Dr Michael Klymkowsky. The following plasmids were obtained from Addgene: 3xFLAG-DVL2 (deposited by Dr Jeff Wrana), pRK5-HA-Ubiquitin-WT (deposited by Dr Ted Dawson), sFRP-1-CS2 + (deposited by Dr Randall Moon). We obtained Image clones encoding hLRP5 (Image 9056965), hFZD4 (Image 5199771), hNDP (Image 5179578) and subcloned the coding sequences into pcDNA3.3 TOPO
} (Invitrogen) using PCR. Constructs contained a HSV-1 glycoprotein D signal sequence (ss) as indicated. pcDNA3.3 ss.V5-FZD4 (aa37-537), pcDNA3.3 ss.FLAG-AP-hNorrin (aa25-133), pAPtag5 ss.AP-hNorrin-mycHis (aa25-133), pcDNA3.3 ss.3xFLAG-hLRP5 (aa32-1615), pcDNA3.3 ss.HA-hLRP5 (aa32-1615) pcDNA3.3 hLRP5 (aal-1615), pcDNA3.3 hWNT3A, and pcDNA3.3 HAhTSPAN12 were verified to be fully functional in TOPFlash assays. pcDNA3.3 V5-FZD4 K13R was generated by subcloning a DNA fragment obtained by gene synthesis (Biobasic), in which all intracellular lysine resides were mutated to arginine, pcDNA3.3 V5-FZD4 K12R (with intact lysine in position 499) and pcDNA3.3 V5-FZD4 M157V were generated by site directed mutagenesis (Quikchange, Stratagene).

Cell culture. 293T cells (ATCC CRL-3216), HeLa cells (ATCC CCL-2), or bEND.3 cells (ATCC CRL-2299 (ref. 53)), were cultured in high glucose DMEM with $10 \%$ FBS (full DMEM) at $37^{\circ} \mathrm{C}$ in the presence of $5 \% \mathrm{CO}_{2}$. For maintenance, cells were split 1:6 at near confluence using $0.05 \%$ Trypsin-EDTA. Mycoplasma contamination was excluded by PCR.

Generation of conditioned medium. The concentration of NDP in the conditioned medium (CM) was significantly increased when NDP was fused to AP. Both FLAG-AP-NDP and AP-NDP-mycHis constructs/CM were fully functional in TOPFlash assays, but allowed for different modes of detection (the detection of FLAG via the M2 antibody is more sensitive than the detection of myc with the $9 \mathrm{E} 10$ antibody). AP-NDP CM was generated by transient transfection of 293T cells for 3-4 days. Acidity was controlled by adding sterile $1 \mathrm{M}$ HEPES $\mathrm{pH}$ 8.0. CM was filtered and stored at $4{ }^{\circ} \mathrm{C}$ or frozen. WNT5A CM was generated from L Wnt-5A cells (ATCC CRL-2814) stably expressing WNT5A. Cells were maintained in the presence of G418 and CM was collected twice for 2 days without G418.

Immunoblotted WNT5A was probed using rabbit anti WNT5A antibody (1:1,000, Cell Signaling, \#2392).

Stimulation of endocytosis. Twenty thousand HeLa cells were seeded into 8-well chamber slides (Labtek II, Nunc) and transfected 3-6h later with a total of $160 \mathrm{ng}$ DNA (typically: $25 \mathrm{ng}$ V5-FZD4, $50 \mathrm{ng}$ LRP5, 50 ng HA-TSPAN12, $35 \mathrm{ng}$ empty vector using TransIT LTI (Mirus). $36 \mathrm{~h}$ later, cells were placed on ice and incubated for $1 \mathrm{~h}$ with ice cold FLAG-AP-NDP or AP-NDP-mycHis CM. For quantitative NDP internalization assays CM was diluted 1:10 with full DMEM. Cells were washed once with ice cold full DMEM and placed in a tissue culture incubator at $37^{\circ} \mathrm{C}$ for $30 \mathrm{~min}$ or the otherwise indicated period of time. Subsequently, cells were washed one time for $3 \mathrm{~min}$ at RT with $150 \mathrm{mM} \mathrm{NaCl}, 50 \mathrm{mM}$ glycine, $\mathrm{pH} 2.4$, and then rinsed twice with PBS before fixation with $10 \% \mathrm{NBF}$ for $5 \mathrm{~min}$ at RT. Where endocytosed FZD4 was visualized by FZD4 pre-labelling ('antibody feeding'), intact cells on ice were incubated with both NDP CM and ms anti V5 (Serotec, 1:2,000) on ice for $1 \mathrm{~h}$, then internalization was triggered at $37^{\circ} \mathrm{C}$ for $30 \mathrm{~min}$. Alternatively, FZD4 endocytosis was triggered with prewarmed WNT5A CM and $1 \mu \mathrm{M}$ PMA (from a $1,600 \times$ stock in DMSO) for $40 \mathrm{~min}$ in a cell culture incubator at $37^{\circ} \mathrm{C}$ (ref. 40). Control cells were incubated with medium containing the same concentration of DMSO. Cells were washed with PBS and fixed for immuno-staining.

Immunofluorescence staining and confocal imaging of cultured cells. After fixation with $10 \% \mathrm{NBF}$ for $10 \mathrm{~min}$ at RT, cells were rinsed twice with PBS and blocked/ permeabilized with PBS, $5 \%$ goat serum, $0.1 \%$ Triton X-100 for $20 \mathrm{~min}$ at RT. Cells were incubated with primary antibodies in blocking buffer for $1 \mathrm{~h}$ at RT, washed three times with PBS, $0.1 \%$ Triton X-100, and incubated with secondary antibody in blocking buffer for $1 \mathrm{~h}$ at RT. After staining, cells were rinsed three times in PBS, $0.1 \%$ Triton X-100 and mounted using Fluoromount G. When two mouse (ms) primary antibodies were used, a three-step protocol was used, that is, the third step was incubation with a ms primary antibody directly conjugated to a fluorophore (for example, ms anti FLAG to detect NDP in the $555 \mathrm{~nm}$ channel, combined with ms V5-Alexa-488 staining as a third step, to detect FZD4). In some experiments triple immunostain was performed using secondary antibodies conjugated to Alexa 488, Alexa 555, and Alexa 647. Primary antibodies were: ms anti V5 (1:2,000, Serotec, clone SV5-PK1, in some experiments this antibody was conjugated to Alexa 488 and used at 1:200), ms anti FLAG (1:1,000, Sigma, clone M2), rat anti HA (1:500, Roche, clone 3F10), mouse anti myc (1:1,000, Thermo, clone 9E10). The following rabbit antibodies were from Cell Signaling: anti myc (1:200, clone 71D10) EEA1 (1:200, clone C45B10), RAB5 (1:200, clone C8B1), RAB7 (1:100, clone D95F2), RAB11 (1:200, clone D4F5), LAMP1 (1:200, clone D2D11). Fluorescence was visualized at RT using a Zeiss LSM510 $(63 \times$ oil $)$ or a Nikon A1 $(100 \times$ oil $)$ confocal laser scanning system. Co-localization analysis was performed using the Pearson's correlation coefficient method in NIS-Elements. NDP positive puncta were quantified using NIS-Elements.

CRISPR/Cas9-mediated gene targeting. HeLa cells were transfected with plasmids encoding Cas9 and sgRNAs (deposited by Dr Feng Zhang: addgene \#42230, co-transfected with GFP, or \#62988, selected with puromycin). Individual colonies derived from single cells were picked and expanded. HeLa cell clones were screened by genomic PCR. PCR products were analyzed after blunt ligation into pJet2.1 by sequencing of 10 individual bacterial clones. Clones were identified as 
positive if targeted alleles were null alleles and if a maximum of two types of targeted alleles was found. sgRNA sequences are shown in Supplementary Figures.

TOPFlash luciferase assay. $293 \mathrm{~T}$ cells were seeded by dispensing $0.4 \mathrm{ml}$ of a $300 \mathrm{~K} \mathrm{ml}^{-1}$ cell suspension into 48 -well plates. $3-6 \mathrm{~h}$ later cells were transfected by combining $20 \mu \mathrm{l}$ Optimem with $160 \mathrm{ng}$ DNA per well and $20 \mu \mathrm{l}$ Optimem with $0.32 \mu \mathrm{l}$ of Trans-IT LT1 (Mirus). After 15 min complex formation at RT the transfection mix was added to the cells. Per well, transfections contained the following plasmids: 4 ng FZD4, 8 ng LRP5, 8 ng pcDNA 3.3 HA-TSPAN12 (or 8 ng pcDNA 3.3 EGFP as control), and 140 ng of reporter mix. 20 ng Vps 4 b was co-transfected in some experiments. Reporter mix had a concentration of $1 \mathrm{mg} \mathrm{ml}^{-1}$ and was composed of TOPFlash plasmid, CMV-Renilla, and Lef1 in a ratio of 7:2:1. Six hours after transfection, cells were stimulated with $125 \mathrm{ng} \mathrm{ml}^{-1}$ recombinant NDP (R + D Systems) for 12-16h. Dual-Glo luciferase assays (Promega) were performed in white, flatbottom, non-treated polystyrene 96-well plates following the manufacturer's instructions. IWP-2 was used at a final concentration of $5 \mu \mathrm{M}$ from cell seeding onward. In experiments where dynasore was added, cells were allowed to express for $17 \mathrm{~h}$, were then grown with dynasore for $1 \mathrm{~h}$ and subsequently with NDP for additional $4 \mathrm{~h} .20 \mathrm{mM}$ or $5 \mathrm{mM}$ dynasore stock solutions in DMSO were diluted 1:500 into a 48-well containing $500 \mu \mathrm{l}$ medium, DMSO in the same dilution was added to control wells. Unless otherwise noted, data were analyzed by calculating the ratio of firefly/renilla luciferase signals and normalizing the data to the data point shown on the left of each bar graph.

Animals. All animal use for this study was approved by the Institutional Animal Care and Use Committee (IACUC) at the University of Colorado Boulder. Male or female animals on a C57BL/6J background were used for experiments at postnatal ages P10 or P19. $\operatorname{Tg}\left(\mathrm{Cdh} 5\right.$-cre/ERT2)1Rha mice were provided by Dr. Ralf Adams ${ }^{47}$ and genotyped by PCR using the following primer set: $5^{\prime}$-GCCTGCATTACCGG TCGATGC-3' $/ 5^{\prime}$-CAGGGTGTTATAAGCAATCCC-3'. Cre-activatable Vps4b EQ mice were generated by a knock-in strategy into the Rosa26 locus. Mouse Vps4bEQ cDNA with MycHis tag and bovine growth hormone polyadenylation sequence was amplified from pEGFP-N1-mVps4b-EQ-MycHis plasmid (gift from Dr Phyllis Hanson) and subcloned into NotI/EcoRI sites of transfer vector pMAT1. The Vps4b EQ sequence with pMAT1 encoded portions of the CAG promoter and lox-STOPlox sequence was subcloned into the MreI and EcoRI sites of pHW249 targeting vector (pMAT1 and pHW249 were kindly provided by Dr Jeremy Nathans). The targeting construct was linearized with KpnI before electroporation into EC7.1 ES cells in the University of Colorado Anschutz Medical Campus gene targeting core. Positive ES cell clones were identified by PCR using primers upstream and downstream of the $5^{\prime}$ arm (5'-AAGAAGAGGCTGTGCTTTG-3' $5^{\prime} / 5^{\prime}$-TTGGCGTT ACTATGGGAAC- $3^{\prime}$ ) as well as using primers upstream and downstream of the $3^{\prime}$ arm (5'-GAACCAGCTGGGGCTCGACTAGAG-3'/5'-CAAGCACTGTCCTGTCC TCAAG- $\left.3^{\prime}\right)$. Selected positive clones were karyotyped and two correct clones were injected into blastocyst to generate chimeric mice. Chimeric mice were crossed with C57BL/6J mice and genotyped for the presence of a $483 \mathrm{bp} \mathrm{Vps} 4 \mathrm{~b}$ amplicon using the primers

5'-TGGAGATAAACTCTTGGAGCC-3'/5'-CCGGAATTCTCCGCCTCAGA AGCCATAGAGC-3'. No randomization or blinding was used in animal studies. Three or more animals per genotype were analyzed.

Preparation and administration of tamoxifen. Tamoxifen (Sigma) solutions were freshly prepared at $1 \mathrm{mg} \mathrm{ml}^{-1}$ in sterile corn oil (Sigma) the day before each injection using a nutator to facilitate dissolution overnight at RT. Solutions were sterile filtered before injection. $50 \mu \mathrm{l} \mathrm{of} 1 \mathrm{mg} \mathrm{ml}^{-1}$ tamoxifen in corn oil was administrated by intraperitoneal injection at P6 and P7 for retinal analysis at P10. For the analysis of P19 brains, $100 \mu \mathrm{l} 20 \mathrm{mg} \mathrm{ml}^{-1}$ tamoxifen was injected at P16 and P17.

Immunofluorescence staining of tissue. P19 deeply anesthetized mice were transcardially perfused with $20 \mathrm{ml} 0.3 \mathrm{mg} \mathrm{ml}^{-1}$ Sulfo-NHS-Biotin (Thermo 21335) in PBS and $10 \mathrm{ml} 2.5 \%$ NBF. Saggital brains sections were collected at 50 or $150 \mu \mathrm{m}$ thickness using a cryostat or vibratome, respectively. Tissues were fixed for $3 \mathrm{~h}$ in $10 \%$ neutral buffered formalin (NBF) on ice. For the generation of retinal sections, the cornea of fixed eyeballs was incised before specimens were cryoprotected in $30 \%$ sucrose, $\mathrm{PBS}$ at $4{ }^{\circ} \mathrm{C}$ overnight. Specimens were embedded in O.C.T. compound (Tissue-Tek), and rapidly frozen. PBS, $0.1 \%$ Triton X-100 (PBST) with $5 \%$ goat serum was used for blocking for $40 \mathrm{~min}$ at RT, primary antibodies diluted in blocking buffer were applied overnight at $4{ }^{\circ} \mathrm{C}$. Slides were washed with $0.1 \%$ PBST 3 times for $2 \mathrm{~min}$. Secondary antibody in blocking buffer was applied for $1 \mathrm{~h}$ at RT, slides were washed 3 times for $2 \mathrm{~min}$ in $0.1 \%$ PBST before mounting. For whole mount retina immunostaining ${ }^{54}$, tissues were fixed for $3 \mathrm{~h}$ in $10 \%$ neutral buffered formalin (NBF) on ice. Retinas were blocked for $2-4 \mathrm{~h}$ at RT in PBST $0.5 \%, 5 \%$ goat serum, and incubated overnight at $4{ }^{\circ} \mathrm{C}$ with primary antibody in blocking buffer. After washing 5 times for $1 \mathrm{~h}$ at RT with $0.5 \%$ PBST, specimens were incubated with secondary antibody and DAPI $\left(1 \mu \mathrm{g} \mathrm{ml}{ }^{-1}\right)$ in blocking buffer at $4{ }^{\circ} \mathrm{C}$. The following primary antibodies and lectins were used for this study: GS Isolectin B4 Alexa-488 conjugate 1:100 (Invitrogen, I21411), rat PLVAP 1:200 (BD Biosciences, 550563). Biotin was detected using Alexa-555 coupled
Streptavidin (1:1,000, Invitrogen). Fluorescence was visualized using a Zeiss LSM510 confocal laser scanning system or Leica DM IL epifluorescent microscope.

Ubiquitination assay. HeLa CCL-2 cells in $10 \mathrm{~cm}^{2}$ dishes were co-transfected with $3 \mu \mathrm{g}$ of HA-Ubiquitin, $1 \mu \mathrm{g}$ of V5-FZD4 (wild type, V5-FZD4-K12R, or V5-FZD4-K13R), and $5 \mu \mathrm{g}$ of EGFP plasmids using $54 \mu \mathrm{l} \mathrm{of} 1 \mathrm{mg} \mathrm{ml}^{-1}$ linear PEI, MW 25,000 (Polysciences). After 20-24h, cells were placed on ice and growth medium was removed. Samples were incubated for $1 \mathrm{~h}$ on ice with $5.5 \mathrm{ml}$ FLAG-AP-NDP conditioned medium (CM) or growth medium, each supplemented with $1 \mu \mathrm{g}$ ms anti-V5 (AbD Serotec). Cells were then incubated at $37^{\circ} \mathrm{C}$ for 20 min to induce internalization. CM was removed and cells were washed twice with ice cold 1x PBS. Cells were then lysed with $4 \mathrm{ml}$ lysis buffer $(150 \mathrm{mM} \mathrm{NaCl}$, $50 \mathrm{mM}$ Tris pH 8.0, $1 \mathrm{mM}$ EGTA, 1\% NP40, 0.1\% N-dodecyl-beta-D-maltoside, $3 \mathrm{mM} \mathrm{MgCl}_{2}$, DNAsel 80 units per $\mathrm{ml}$ (Roche), and $1 \times$ complete ultra EDTA-free protease inhibitor cocktail (Roche)), on ice for $40 \mathrm{~min}$ with gentle shaking. Cell debris and unsolubilized material were pelleted at $20,000 \mathrm{~g}$ centrifugation at $4{ }^{\circ} \mathrm{C}$ for $45 \mathrm{~min}$. For the analysis of input from each sample, $104 \mu \mathrm{l}$ of the supernatant were removed and combined with $40 \mu \mathrm{l} 4 \times$ LDS sample buffer (Invitrogen) and $16 \mu \mathrm{l}$ 1M DTT. $3.75 \mathrm{ml}$ of remaining supernatant were incubated with $30 \mu \mathrm{l}$ ProteinA/G beads (Pierce) at $4{ }^{\circ} \mathrm{C}$ for $45 \mathrm{~min}$ with gentle rocking. Samples were transferred into spin columns with screw cap (Pierce), which were attached to a vacuum manifold (Qiagen) and washed 5 times with $0.7 \mathrm{ml}$ wash buffer $(150 \mathrm{mM} \mathrm{NaCl}, 50 \mathrm{mM}$ Tris $\mathrm{pH} 8.0,1 \mathrm{mM}$ EGTA, $1 \% \mathrm{NP} 40,3 \mathrm{mM} \mathrm{MgCl} 2$ ) at $\mathrm{RT}$ by applying vacuum. V5-FZD4 and associated proteins were eluted from the beads by incubating the plugged and capped columns with $120 \mu \mathrm{l} 1 \times$ LDS sample buffer (Invitrogen) containing $100 \mathrm{mM}$ DTT for $20 \mathrm{~min}$ at $45^{\circ} \mathrm{C}$ in a thermomixer (Eppendorf) with rapid shaking. Eluates were spun through the column into collection tubes. $45 \mu \mathrm{l}$ of the eluate were loaded onto SDS-PAGE gels. Anti-HA-HRP (1:1,000, Roche), anti-V5-HRP (1:10,000, AbD Serotec), and anti-FLAG-HRP (1:1,000, Sigma) were used to probe immunoblots.

Software and figure preparation. Zeiss Zen 2011, Nikon NIS-Elements, ImageJ, Microsoft Office 2016 and Adobe Photoshop/Illustrator CS6 were used for data analysis and figure preparation. Full scans of immunoblots are shown in Supplementary Fig. 11.

Statistics. Averages were calculated from triplicates. Groups were compared using a two-tailed, unpaired Student's $t$-test. $P$-values $<0.05$ were considered significant.

Data availability. All relevant data are available from the authors.

\section{References}

1. Clevers, H. \& Nusse, R. Wnt/beta-catenin signaling and disease. Cell 149, 1192-1205 (2012).

2. Liebner, S. et al. Wnt/beta-catenin signaling controls development of the blood-brain barrier. J. Cell Biol. 183, 409-417 (2008).

3. Stenman, J. M. et al. Canonical Wnt signaling regulates organ-specific assembly and differentiation of CNS vasculature. Science 322, 1247-1250 (2008).

4. Daneman, R. et al. Wnt/beta-catenin signaling is required for CNS, but not non-CNS, angiogenesis. Proc. Natl Acad. Sci. USA 106, 641-646 (2009).

5. Wang, Y. et al. Norrin/Frizzled4 signaling in retinal vascular development and blood brain barrier plasticity. Cell 151, 1332-1344 (2012).

6. Zhou, Y. et al. Canonical WNT signaling components in vascular development and barrier formation. J. Clin. Invest. 124, 3825-3846 (2014).

7. Zhao, Z., Nelson, A. R., Betsholtz, C. \& Zlokovic, B. V. Establishment and dysfunction of the blood-brain barrier. Cell 163, 1064-1078 (2015).

8. Ye, X., Wang, Y. \& Nathans, J. The Norrin/Frizzled4 signaling pathway in retinal vascular development and disease. Trends Mol. Med. 16, 417-425 (2010).

9. Junge, H. J. et al. TSPAN12 regulates retinal vascular development by promoting Norrin- but not Wnt-induced FZD4/beta-catenin signaling. Cell 139, 299-311 (2009).

10. Chang, T. H. et al. Structure and functional properties of Norrin mimic Wnt for signalling with Frizzled4, Lrp5/6, and proteoglycan. Elife 4, e06554 (2015).

11. Shen, G. et al. Structural basis of the Norrin-Frizzled 4 interaction. Cell Res. $\mathbf{2 5}$, 1078-1081 (2015).

12. Xu, Q. et al. Vascular development in the retina and inner ear: control by Norrin and Frizzled-4, a high-affinity ligand-receptor pair. Cell 116, 883-895 (2004).

13. Luhmann, U. F. et al. Role of the Norrie disease pseudoglioma gene in sprouting angiogenesis during development of the retinal vasculature. Invest. Ophthalmol. Vis. Sci. 46, 3372-3382 (2005).

14. Ye, X. et al. Norrin, frizzled-4, and Lrp5 signaling in endothelial cells controls a genetic program for retinal vascularization. Cell 139, 285-298 (2009).

15. Xia, C. H. et al. A model for familial exudative vitreoretinopathy caused by LPR5 mutations. Hum. Mol. Genet. 17, 1605-1612 (2008). 
16. Gilmour, D. F. Familial exudative vitreoretinopathy and related retinopathies. Eye 29, 1-14 (2015).

17. MacDonald, B. T. \& He, X. Frizzled and LRP5/6 receptors for Wnt/beta-catenin signaling. Cold Spring Harb. Perspect. Biol. 4, a007880 (2012).

18. Bilic, J. et al. Wnt induces LRP6 signalosomes and promotes dishevelleddependent LRP6 phosphorylation. Science 316, 1619-1622 (2007).

19. Pan, W. et al. Wnt3a-mediated formation of phosphatidylinositol 4,5-bisphosphate regulates LRP6 phosphorylation. Science 321, 1350-1353 (2008).

20. Yu, A., Xing, Y., Harrison, S. C. \& Kirchhausen, T. Structural analysis of the interaction between Dishevelled2 and clathrin AP-2 adaptor, a critical step in noncanonical Wnt signaling. Structure 18, 1311-1320 (2010).

21. Kim, I. et al. Clathrin and AP2 are required for PtdIns(4,5)P2-mediated formation of LRP6 signalosomes. J. Cell Biol. 200, 419-428 (2013).

22. Hagemann, A. I. et al. In vivo analysis of formation and endocytosis of the Wnt/beta-catenin signaling complex in zebrafish embryos. J. Cell Sci. 127, 3970-3982 (2014)

23. Jiang, Y., He, X. \& Howe, P. H. Disabled-2 (Dab2) inhibits Wnt/beta-catenin signalling by binding LRP6 and promoting its internalization through clathrin. EMBO J. 31, 2336-2349 (2012).

24. Yamamoto, H., Sakane, H., Yamamoto, H., Michiue, T. \& Kikuchi, A. Wnt3a and Dkk1 regulate distinct internalization pathways of LRP6 to tune the activation of beta-catenin signaling. Dev. Cell 15, 37-48 (2008).

25. Yamamoto, H., Komekado, H. \& Kikuchi, A. Caveolin is necessary for Wnt-3a-dependent internalization of LRP6 and accumulation of beta-catenin. Dev. Cell 11, 213-223 (2006).

26. Niehrs, C. The complex world of WNT receptor signalling. Nat. Rev. Mol. Cell. Biol. 13, 767-779 (2012).

27. Feng, Q. \& Gao, N. Keeping Wnt signalosome in check by vesicular traffic. J. Cell. Physiol. 230, 1170-1180 (2015).

28. Dobrowolski, R. \& De Robertis, E. M. Endocytic control of growth factor signalling: multivesicular bodies as signalling organelles. Nat. Rev. Mol. Cell. Biol. 13, 53-60 (2012)

29. Vinyoles, M. et al. Multivesicular GSK3 sequestration upon Wnt signaling is controlled by p120-catenin/cadherin interaction with LRP5/6. Mol. Cell 53, 444-457 (2014).

30. Taelman, V. F. et al. Wnt signaling requires sequestration of glycogen synthase kinase 3 inside multivesicular endosomes. Cell 143, 1136-1148 (2010).

31. Metcalfe, C. \& Bienz, M. Inhibition of GSK3 by Wnt signalling--two contrasting models. J. Cell Sci. 124, 3537-3544 (2011).

32. Cruciat, C. M. et al. Requirement of prorenin receptor and vacuolar $\mathrm{H}+$-ATPase-mediated acidification for Wnt signaling. Science 327, 459-463 (2010).

33. Mukai, A. et al. Balanced ubiquitylation and deubiquitylation of Frizzled regulate cellular responsiveness to Wg/Wnt. EMBO J. 29, 2114-2125 (2010).

34. de Lau, W., Peng, W. C., Gros, P. \& Clevers, H. The R-spondin/Lgr5/Rnf43 module: regulator of Wnt signal strength. Genes Dev. 28, 305-316 (2014).

35. Hao, H. X. et al. ZNRF3 promotes Wnt receptor turnover in an R-spondin-sensitive manner. Nature 485, 195-200 (2012).

36. Hao, H. X., Jiang, X. \& Cong, F. Control of Wnt receptor turnover by R-spondin-ZNRF3/RNF43 signaling module and its dysregulation in cancer. Cancers 8, 54 (2016).

37. Koo, B. K. et al. Tumour suppressor RNF43 is a stem-cell E3 ligase that induces endocytosis of Wnt receptors. Nature 488, 665-669 (2012).

38. Yu, A. et al. Association of dishevelled with the clathrin AP-2 adaptor is required for Frizzled endocytosis and planar cell polarity signaling. Dev. Cell 12, 129-141 (2007)

39. Nikopoulos, K. et al. Overview of the mutation spectrum in familial exudative vitreoretinopathy and Norrie disease with identification of 21 novel variants in FZD4, LRP5, and NDP. Hum. Mutat. 31, 656-666 (2010).

40. Chen, W. et al. Dishevelled 2 recruits beta-arrestin 2 to mediate Wnt5A-stimulated endocytosis of Frizzled 4. Science 301, 1391-1394 (2003).

41. Tauriello, D. V. et al. Wnt/beta-catenin signaling requires interaction of the Dishevelled DEP domain and C terminus with a discontinuous motif in Frizzled. Proc. Natl Acad. Sci. USA 109, E812-E820 (2012).

42. Umbhauer, M. et al. The C-terminal cytoplasmic Lys-thr-X-X-X-Trp motif in frizzled receptors mediates $\mathrm{Wnt} /$ beta-catenin signalling. EMBO J. 19, 4944-4954 (2000).

43. Boonstra, F. N. et al. Clinical and molecular evaluation of probands and family members with familial exudative vitreoretinopathy. Invest. Ophthalmol. Vis. Sci. 50, 4379-4385 (2009).

44. Veeman, M. T., Slusarski, D. C., Kaykas, A., Louie, S. H. \& Moon, R. T. Zebrafish prickle, a modulator of noncanonical Wnt/Fz signaling, regulates gastrulation movements. Curr. Biol. 13, 680-685 (2003).
45. Schmidt, O. \& Teis, D. The ESCRT machinery. Curr. Biol. 22, R116-R120 (2012).

46. Bishop, N. \& Woodman, P. ATPase-defective mammalian VPS4 localizes to aberrant endosomes and impairs cholesterol trafficking. Mol. Biol. Cell. 11, 227-239 (2000).

47. Wang, Y. et al. Ephrin-B2 controls VEGF-induced angiogenesis and lymphangiogenesis. Nature 465, 483-486 (2010).

48. Langton, P. F., Kakugawa, S. \& Vincent, J. P. Making, exporting, and modulating Wnts. Trends Cell. Biol. 26, 756-765 (2016).

49. Deng, C. et al. Multi-functional norrin is a ligand for the LGR4 receptor. J. Cell Sci. 126, 2060-2068 (2013).

50. Zhou, Y. \& Nathans, J. Gpr124 controls CNS angiogenesis and blood-brain barrier integrity by promoting ligand-specific canonical wnt signaling. Dev. Cell 31, 248-256 (2014)

51. Acebron, S. P. \& Niehrs, C. beta-catenin-independent roles of Wnt/LRP6 signaling. Trends Cell. Biol. 26, 956-967 (2016).

52. Pitulescu, M. E. \& Adams, R. H. Regulation of signaling interactions and receptor endocytosis in growing blood vessels. Cell Adhes. Migr. 8, 366-377 (2014).

53. Montesano, R. et al. Increased proteolytic activity is responsible for the aberrant morphogenetic behavior of endothelial cells expressing the middle $\mathrm{T}$ oncogene. Cell 62, 435-445 (1990).

54. Johnson, V., Xiang, M., Chen, Z. \& Junge, H. J. Neurite mistargeting and inverse order of intraretinal vascular plexus formation precede subretinal vascularization in Vldlr mutant mice. PLoS ONE 10, e0132013 (2015).

\section{Acknowledgements}

The authors would like to thank Dr J. Nathans for providing pMAT1 and pHW249 constructs for the generation of Cre-activatable alleles in the Rosa26 locus. We also thank Drs J. Tyler, J. Orth, and C. English in the MCDB light microscopy core facility for assistance in confocal imaging, as well as M. Stoltz and A. Fosdick in the animal facility for their support, and Drs L. Sun and H. Wang for assistance in CRISPR/Cas9 mediated gene targeting. This work was supported by ACS IRG \#57-001-53 from the American Cancer Society, the Boettcher Foundation Webb-Waring Biomedical Research Award (to H.J.J.), and a grant from the NIH (R01EY024261 to H.J.J.)

\section{Author contributions}

C.Z., M.B.L., L.K., Z.C. and H.J.J. designed experiments. C.Z., M.B.L., L.K., L.A.L. and H.J.J. conducted experiments and analysed data. C.Z., Z.C. and H.J.J. wrote the manuscript. All authors edited the manuscript.

\section{Additional information}

Supplementary Information accompanies this paper at http://www.nature.com/ naturecommunication

Competing interests: The authors declare no competing financial interests.

Reprints and permission information is available online at http://npg.nature.com/ reprintsandpermissions/

How to cite this article: Zhang, C. et al. Norrin-induced Frizzled4 endocytosis and endo-lysosomal trafficking control retinal angiogenesis and barrier function. Nat. Commun. 8, 16050 doi: 10.1038/ncomms16050 (2017).

Publisher's note: Springer Nature remains neutral with regard to jurisdictional claims in published maps and institutional affiliations.

Open Access This article is licensed under a Creative Commons Attribution 4.0 International License, which permits use, sharing, adaptation, distribution and reproduction in any medium or format, as long as you give appropriate credit to the original author(s) and the source, provide a link to the Creative Commons license, and indicate if changes were made. The images or other third party material in this article are included in the article's Creative Commons license, unless indicated otherwise in a credit line to the material. If material is not included in the article's Creative Commons license and your intended use is not permitted by statutory regulation or exceeds the permitted use, you will need to obtain permission directly from the copyright holder. To view a copy of this license, visit http://creativecommons.org/ licenses/by/4.0/

(C) The Author(s) 2017 